\title{
Reduction of applicator displacement in MR/CT-guided cervical cancer HDR brachytherapy by the use of patient hover transport system
}

\author{
Megan Andrew!, Yusung Kim, PhDl,2, Timothy Ginader, MS², Brian J. Smith, PhD, Wenging Sun, MD, PhD 1,2, \\ Dongxu Wang, PhD',2 \\ 'Department of Radiation Oncology, University of lowa Hospitals and Clinics, ${ }^{2}$ Holden Comprehensive Cancer Center, University of lowa \\ Hospitals and Clinics, ${ }^{3}$ Department of Biostatistics, University of lowa, lowa City, IA, USA
}

\begin{abstract}
Purpose: To quantify the reduction of relative displacement between the implanted intracavitary applicator and the patient bony anatomy, due to the use of a hover transport system during the patient transports between the imaging table and the treatment table.

Material and methods: The displacement of the applicator inside the patient was measured by comparing the distance between the tip of the tandem and the pubic bone on X-ray radiography images taken before and after moving a patient to magnetic resonance/computed tomography imaging. Displacements were evaluated for 27 fractions of treatment using hover transport and 185 fractions of treatment using manual transport.

Results: The use of hover transport system reduced the percentage of fractions with displacements greater than $5 \mathrm{~mm}$ from $22.7 \%$ to $7.4 \%$. The reduction of applicator displacement using hover transport is statistically significant, compared to the manual transport method ( $p$-value 0.0086; mean displacement $3.41 \mathrm{~mm}$ [95\% CI: 2.96-3.97] for manual transport, and $2.27 \mathrm{~mm}$ [95\% CI: 1.71-2.97] for hover transport fractions).

Conclusions: This study indicates that the hover transport system is effectively reducing displacement between tandem and patient bony anatomy during patient transports. The potential improvement in dosimetric accuracy due to this reduction warrants further study.

Key words: applicator displacement, cervical cancer, HDR, MRI-guided.

\section{Purpose}

The American Cancer Society estimates that in 2016 there will be 12,990 new cases of invasive cervical cancer diagnosed, and approximately 4,120 women will die from cervical cancer [1]. Since 2000, the American Brachytherapy Society has recommended the use of and provided guidelines for high-dose-rate (HDR) brachytherapy in cervical cancer treatment [2]. This recommendation came after several patterns of care studies found that the combined use of external beam radiation therapy with brachytherapy, in treatment, decreased recurrence and complications in patients $[2,3,4,5,6,7]$. HDR brachytherapy has since become a major part of treatment for cervical cancer stages I-IV because it allows for the application of a high radiation dose to the cervix, but significantly lower doses to the bladder, sigmoid, small bowel, and rectum [8], compared to local external beam radiation therapy. It is recommended by Groupe Européen de Curiethérapie and the European Society for Radiotherapy \& Oncology (GEC-ESTRO) that the patient undergo magnetic resonance imaging (MRI) or computed tomography (CT) imaging during HDR brachytherapy, so that the treatment plan can be most accurately created based on patient anatomy and the location and size of implanted brachytherapy applicators $[8,9,10,11]$. In this MR/CT-guided HDR brachytherapy, patients must be moved to and from MR/CT scanner while the brachytherapy applicators, most commonly tandem and ovoids (T\&O) or tandem and ring, are implanted inside their body. It has been found that, during a manual transport of a sedated patient, the $\mathrm{T} \& \mathrm{O}$ applicator can become displaced, and this may lead to varied radiation dose to certain reference points [12]. GEC-ESTRO [10,11,12,13] reports around 5\% dose-volume error to both target volume and organ-at-risk for each $1 \mathrm{~mm}$ error in applicator reconstruction, when a plan is optimized based upon 3D image. One can expect similar dose error due to applicator displacement for 3D image-

Address for correspondence: Dongxu Wang, PhD, Department of Radiation Oncology, University of Iowa Received: 11.10.2017 Hospitals and Clinics, 200 Hawkins Drive, Radiation Oncology, Iowa City, IA 52242, USA, 
guided brachytherapy plans. Grigsby et al. investigated the inter-fractional tandem displacement between multiple insertions, and found that about $1.2 \mathrm{~cm}$ displacement of tandem in the caudal-cranial direction, therefore $1.2 \mathrm{~cm}$ displacement of geometric point $\mathrm{A}$, can lead to up to $35 \%$ of point dose difference in the same anatomical location, when a point A based-plan was generated on orthogonal $\mathrm{X}$-rays [14]. Consequently, if such displacement occurs intra-fractionally, one should expect dose variation of similar magnitude as well as shown by some recent studies $[15,16]$. Some studies have looked into the geometric displacement, anatomic change, and other effect in each fraction of image-guided brachytherapy $[17,18,19,20,21]$. Monitoring of applicator motion relative to patient is therefore important in MR/CT-guided HDR brachytherapy. Efforts should also be made to minimize the disturbance to the inserted or implanted applicator; transport of the patient to and from MR/CT scanner might be such a source of such disturbance.

In June of 2014, our institution began using the Zephyr HDR Patient Positioning and Transfer System (Diacor Inc, Salt Lake City, Utah, USA) in HDR brachytherapy treatments for cervical cancer patients. Prior to that, sedated patients were transported on a white board to and from MR/CT scanner manually by a group of healthcare workers. The dosimetric impact of using the manual transport on white board was reported [22]. Compared to manual patient transport, hover transport was expected to reduce staff injury risk, improve staffing efficiency, and improve clinical workflow. Since the use of the hover transport system, it came to the attention of the clinicians that very large $(>1 \mathrm{~cm})$ intracavitary applicator displacement relative to patient bony anatomy no longer appears in our HDR brachytherapy. While it is intuitive to hypothesize that intracavitary applicator stays more stable during hover transport than during manual transport, no data were collected to prove that.

In this study, we quantify the reduction of relative displacement between the implanted intracavitary applicator and the patient bony anatomy, during HDR brachytherapy treatment for cervical cancer, due to the use of a hover transport system during patient transport.

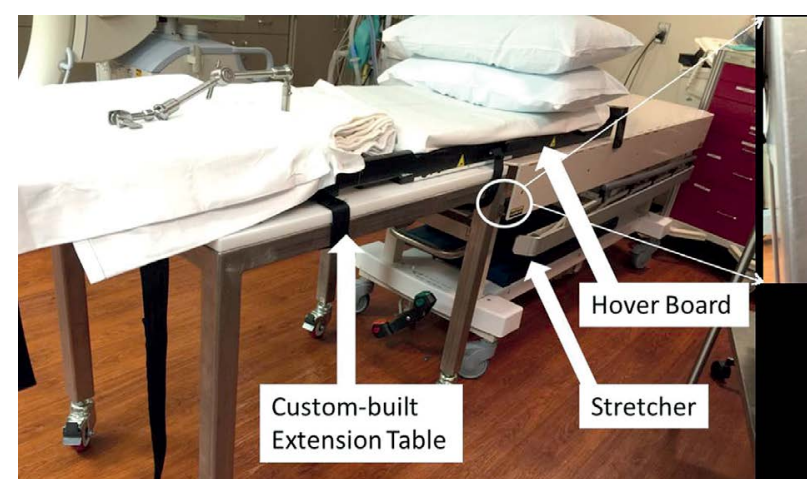

Fig. 1. The hover board, stretcher, and the custom-built extension table, which allows for C-arm radiography. The attachment between the extension table and the stretcher is magnified to show the connection details
We expect these results to show the use of the hover transport system to help the implanted intracavitary applicator stay more stable inside patient.

\section{Material and methods}

\section{Patient selection and clinical procedure}

Six cervical cancer patients, with a total of 27 treatment fractions transported with the hover transport system (Hover), and 51 patients with a total of 185 fractions transported with the manual method (Manual) using a whiteboard specially designed for 3D image-guided HDR brachytherapy, were enrolled in this Institutional Review Board (IRB) - approved study. During each fraction of HDR brachytherapy, the patient was first sedated under general anesthesia on top of either the hover sled for Hover cases or the whiteboard for Manual cases. Then, the Fletcher-Suit-Delclos style T\&O (Varian Medical Systems, Palo Alto, CA, USA) were implanted and packed with gauze packing, while patient legs were on surgical stirrups. Patient legs were put down from stirrups after the tandem and ovoid insertion, and were bound in natural parallel positions by Velcro straps. C-arm (Siemens Medical Solutions USA, Malvern, PA, USA) radiography was then performed, and the resulting image would be referred to as the 'Before Image'. Then, the patient was set up ready for movement, which may take 30 minutes, most of which were spent on disconnecting and connecting anesthesia equipment. For the Manual cases, an applicator-implanted patient along with the whiteboard was then moved to a transport table, then transported to MR or CT scanner, and then moved again to the MR or CT imaging table, where the MR or CT scan was performed. For the Hover cases, the hover transport system is MR conditional, thus an applicator-implanted patient was directly moved to the MR or CT imaging table with a hover sled. The movement to the MR/CT scanner usually took then less than five minutes, as the scanners are just across hallway from the brachytherapy procedure room. MR scan of three sequences (T1, T2 3D, T2 sagittal) took a total of 45 minutes. If it was a CT scan, it took ten minutes or less. After MR or CT imaging, the patient was moved back to the transport table, transported back to the HDR treatment room, and moved back to the treatment table. C-arm radiography imaging was again performed and this resulting image would be referred to as the "After Image". The distance between the tandem and bony anatomy was verified and displacement was measured. The treatment planning and delivery then proceed if the displacement was within tolerance, or by attending physician's discretion. Patient lay on brachytherapy table for forty minutes to one hour, while the brachytherapy plan was being created.

\section{Equipment}

The hover transport system consists of a hover sled, which lifts up 2-3 cm centimeters without a patient when air blows below it, an air blower that attaches to the hover sled, and a stretcher which serves as the base of the hovering sled and the operating bed. The hover transport 


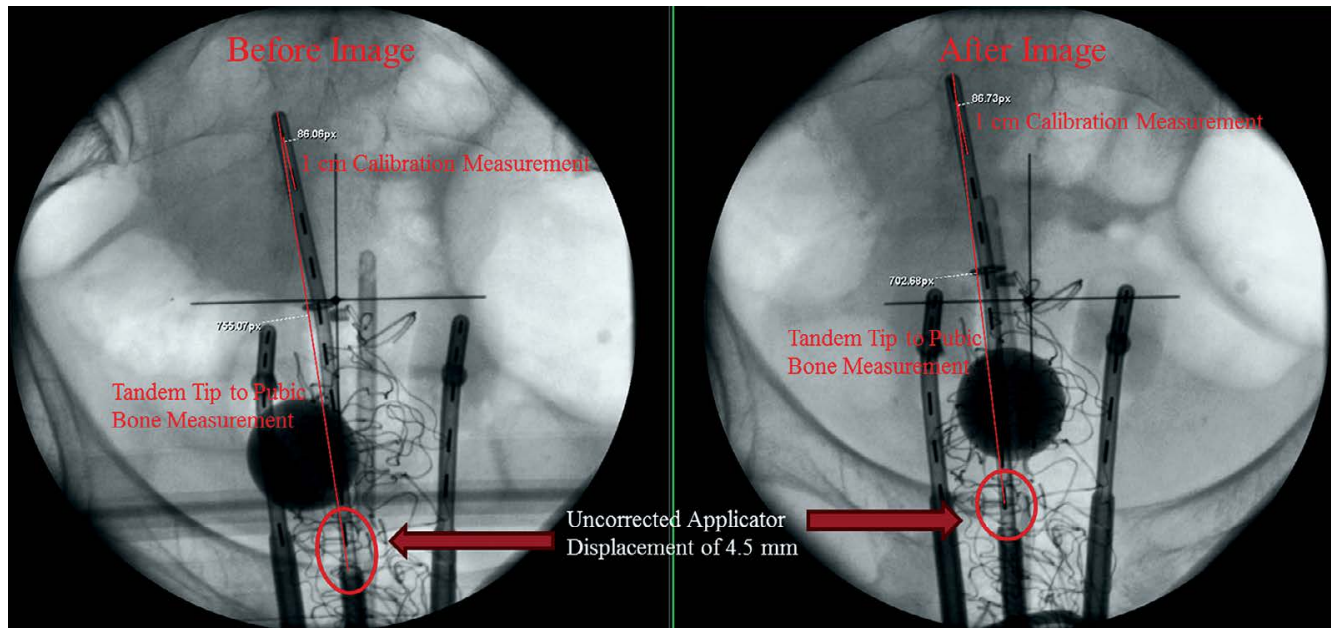

Fig. 2. Example tandem tip to pubic bone measurement and distance calibration on anterior-posterior (C-arm angle: 90 degree) X-ray image acquired before and after moving a patient for MR/CT scan

stretcher does not contain sufficient space underneath to allow for C-arm radiography imaging. To enable C-arm imaging, an extension table was custom made at the authors' institution. Figure 1 shows the attaching mechanism between the extension table and existing hover transport. During C-arm imaging, the patient and hover sled were slid onto the extension imaging table.

The current white board below patient used in the Manual transport was developed after observing considerable applicator displacements during the transfer of an applicator-implanted patient by the use of conventional white board. The white board's lower part is detachable after stirring-up patient's legs, so that an applicator can be implanted without having to move patient's upper body. After an applicator implementation, the lower part is attached, and a patient can be moved to a transfer table with this board.

\section{Displacement measurement}

As shown in Figure 2, the displacement of the implanted $\mathrm{T} \& \mathrm{O}$ applicator during transport was analyzed by comparing the distance between the tip of the tandem and the top of pubic symphysis on anterior-posterior (AP) X-ray radiography images taken before ("Before
Image") and after ("After Image") moving the patient to MR/CT imaging. Radiography pixel distances were measured and then converted to distances in centimeters, using a known $1 \mathrm{~cm}$ distance between tandem dummy seeds (X-ray markers).

The tandem tip and the pubic bones do not usually lie on the same plane perpendicular to the imaging sourcedetector direction; they are projected to the same detector plane. This creates an error in the distance measurements on the AP X-ray images due to X-ray beam divergence. A correction term to compensate for this error was applied to the distance measurements. The derivation and calculation of the correction term is shown in the Appendix.

\section{Statistics}

SAS version 9.4 (SAS Institute, Cary, NC, USA) was used for statistical analysis. For displacement comparison between the Hover and the Manual transport methods, results are obtained from a regression model fit with a generalized estimating equations approach using the gamma distribution for displacement measurements, a log link function for the mean, and a compound symmetry correlation structure for repeated measures.
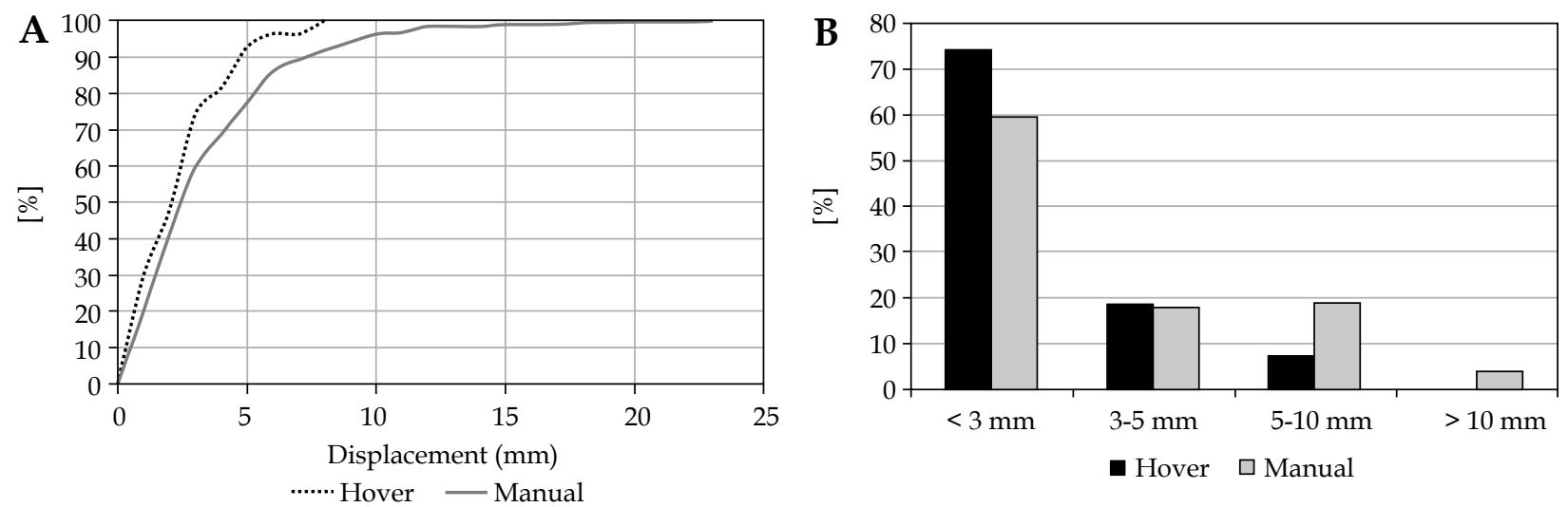

Fig. 3. Frequency of displacements. A) Cumulative frequency. B) Frequency for four displacement ranges 


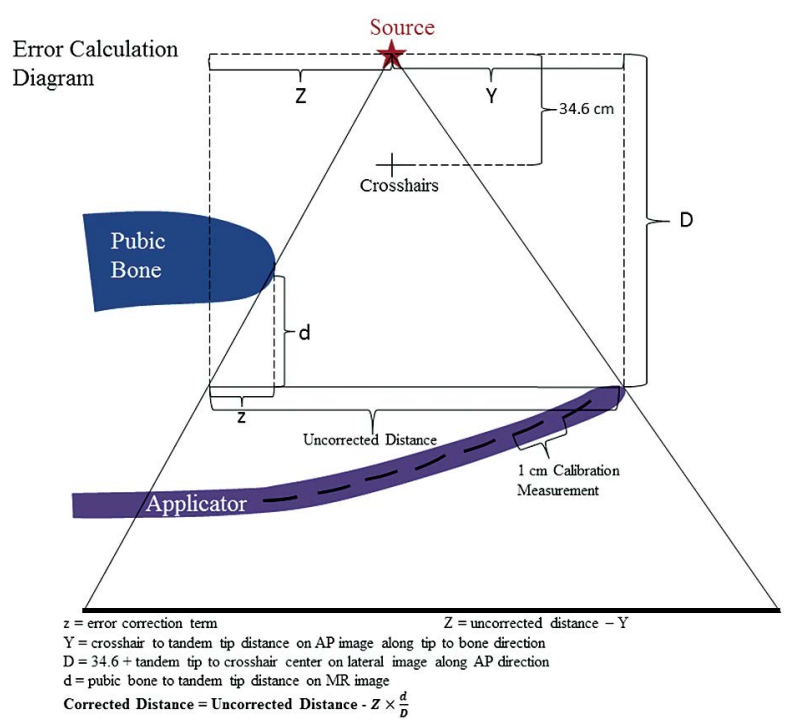

Fig. 4. Correction term calculation diagram. Distances are not to scale
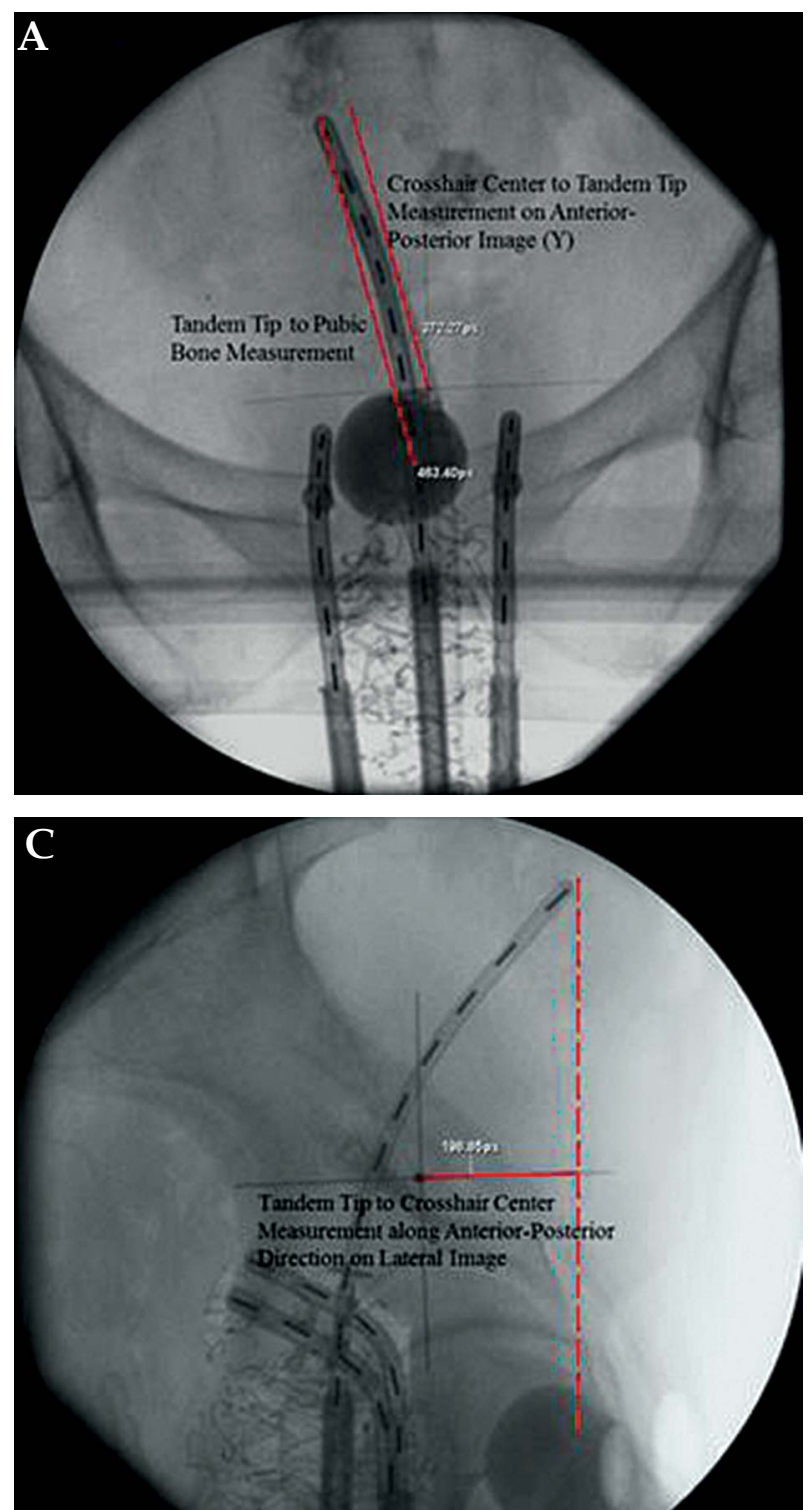

\section{Results}

The cumulative frequency of displacements and the frequency of four displacement ranges $(0-3 \mathrm{~mm}, 3-5 \mathrm{~mm}$, $5-10 \mathrm{~mm}$, and $>10 \mathrm{~mm}$ ) were plotted in Figure 3 . The leftward shift of the cumulative frequency data in Figure 3A shows a reduction of high applicator displacement values with the use of the Hover transport. Figure 3B also shows this trend, as the frequency of the $5-10 \mathrm{~mm}$ and $>10 \mathrm{~mm}$ displacement values are much smaller for the Hover transport than the Manual transport. The use of the Hover transport reduced the percentage of fractions with displacements greater than $5 \mathrm{~mm}$ from $22.7 \%$ to $7.4 \%$, and the percentage of fractions with displacements greater than $10 \mathrm{~mm}$ from $3.78 \%$ to $0.0 \%$. The mean applicator displacement for the Hover transport fractions was $2.27 \mathrm{~mm}$ (95\% CI: 1.71-2.97), and the mean applicator displacement for the Manual transport fractions was $3.41 \mathrm{~mm}$ (95\% CI: 2.96-3.97). The reduction of the applicator displacement with the use of the Hover transport,

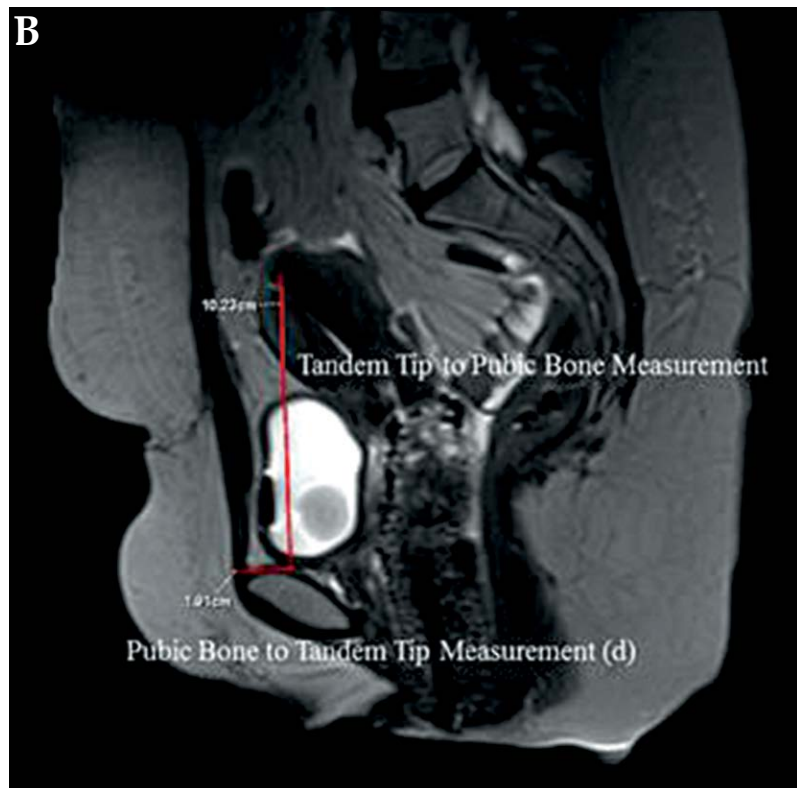

Fig. 5. Distance measurements used in the correction calculation. A) Isocenter (the cross hair) to tandem tip measurement on anterior-posterior (AP) X-ray image along the tandem tip to pubic symphysis direction, used as variable $\mathrm{Y}$ in the correction equation. B) Pubic bone to tandem tip measurement in the AP direction on magnetic resonance image, used as variable $\mathrm{D}$ in the correction equation. C) Tandem tip to isocenter measurement along AP direction on lateral X-ray image, added to $34.6 \mathrm{~cm}$ and used as variable $\mathrm{D}$ in the correction equation 
compared to the Manual transport, was statistically significant with a $p$ value of 0.0086 .

\section{Discussion}

It has been clear to the authors that the use of the Hover system reduces staff injury risk, and the strenuous effort during move and transport for sedated patient. It should be noted that this study measures the displacement of the intrauterine tandem relative to the pubic symphysis, not the uterus or cervix. It is entirely possible that while tandem appears to have moved relative to bones for several millimeters, it still stays intact relative the cervix and uterus. However, without more intensive and more frequent volumetric imaging, constant monitoring of applicator and the tumor is not practically achievable. Nevertheless, an intracavitary applicator to bone displacement based on radiography is still valuable, as lack of displacement relative to bone should indicate lack of displacement relative to uterus and cervix, especially in the superior-inferior direction, where tumor volume uncertainty may exist [23].

Measurement of tandem tip to pubic symphysis distance on $\mathrm{X}$-ray radiography image carries inherent uncertainty. The correction term to account for X-ray divergence improves the accuracy of the measured displacement, although residual errors may remain. Another potential limitation of this study is the low number of treatment fractions available for fractions using the Hover transport, yet the statistical analysis still yielded significant results. The study collected 27 distance measurements on 6 patients transported with the Hover transport system, and 185 measurements on 51 patients transported manually. Although the number of patients might be considered low, a repeated measures statistical approach (generalized estimating equations) was employed to utilize all 212 measurements in the analysis. Since the 212 measurements include repeated observations on patients, they do not carry the same amount of information as individual measurements collected on 212 patients. Nevertheless, based on a low within-patient correlation observed in our data, we estimate that our measurements carry information (effective sample size) equivalent to 189 independent patient measurements. A typical concern of low sample size is low study power to detect an effect. In particular, low power decreases the chances of obtaining a statistically significant result. In this study, the difference between Hover and Manual transport is statistically significant, which cannot be attributed to low power/sample size. The 27 fractions with hover transport may be representative of the population, but further investigation of more fractions would be desirable.

It should be also noted that the operating bed of the original patient hover transport system does not allow the c-arm to rotate through it. The authors' institution made customized extension table (Figure 1) to allow the use of C-arm. The effort spent in such customary work added important quality check capacity to our clinical workflow, justified by the frequently observed intracavitary applicator movement.

\section{Conclusions}

The hover transport system also effectively reduces the displacement of the implanted tandem and ovoids relative to the pubic symphysis. It is not yet clear whether the applicator was displaced along with or separately from the uterus and cervix. The dosimetric effect therefore is pending further investigation.

\section{Appendix}

Due to C-arm X-ray beam divergence, the measured distance between tandem tip and pubic bone, even after distance calibration near the tandem tip, still contains an error, indicated by the distance $z$, as the Figure 4 shows. This error can be accounted for following trigonometry of the imaging geometry. Figure 4 presents how to calculate $z$ based on several measurable distances and known source to isocenter (projected as the cross hair on image) distance of $34.6 \mathrm{~cm}$; Figure 5 shows how some of the distances are measured on the MR (or CT, if CT was used) image and lateral $\mathrm{X}$-ray radiography.

\section{Acknowledgement}

Megan Andrew was supported by Iowa Center for Research by Undergraduate (ICRU).

\section{Disclosure}

Authors report no conflict of interest.

\section{References}

1. Cancer Facts \& Figures 2016. American Cancer Society, Atlanta 2016.

2. Nag S, Erickson B, Thomadsen B et al. The American Brachytherapy Society recommendations for high-dose-rate brachytherapy for carcinoma of the cervix. Int J Radiat Oncol Biol Phys 2000; 48: 201-211.

3. Coia L, Won M, Lanciano R et al. The patterns of care outcome study for cancer of the uterine cervix. Results of the Second National Practice Survey. Cancer 1990; 66: 2451-2456.

4. Hanks GE, Herring DF, Kramer S. Patterns of care outcome studies results of the national practice in cancer of the cervix. Cancer 1983; 51: 959-967.

5. Lanciano RM, Won M, Coia L et al. Pretreatment and treatment factors associated with improved outcome in squamous cell carcinoma of the uterine cervix: a final report of the 1973 and 1978 patterns of care studies. Int J Radiat Oncol Biol Phys 1991; 20: 667-676.

6. Montana GS, Hanlon A, Brickner T et al. Carcinoma of the cervix: patterns of care studies: review of 1978, 1983, and 19881989 surveys. Int J Radiat Oncol Biol Phys 1995; 32: 1481-1486.

7. Montana GS, Martz KL, Hanks GE. Patterns and sites of failure in cervix cancer treated in the USA in 1978. Int J Radiat Oncol Biol Phys 1991; 20: 87-93.

8. Haie-Meder C, Pötter R, van Limbergen E et al. Recommendations from Gynaecological (GYN) GEC-ESTRO Working Group (I): concepts and terms in 3D image based 3D treatment planning in cervix cancer brachytherapy with emphasis on MRI assessment of GTV and CTV. Radiother Oncol 2005; 74: 235-245.

9. Dimopoulos JC, Petrow P, Tanderup K et al. Recommendations from Gynaecological (GYN) GEC-ESTRO Working 
Group (IV): Basic principles and parameters for MR imaging within the frame of image based adaptive cervix cancer brachytherapy. Radiother Oncol 2012; 103: 113-122.

10. Hellebust TP, Kirisits C, Berger D et al. Recommendations from Gynaecological (GYN) GEC-ESTRO Working Group: considerations and pitfalls in commissioning and applicator reconstruction in 3D image-based treatment planning of cervix cancer brachytherapy. Radiother Oncol 2010; 96: 153-160.

11. Pötter R, Haie-Meder C, van Limbergen $E$ et al. Recommendations from gynaecological (GYN) GEC ESTRO Working Group (II): Concepts and terms in 3D image-based treatment planning in cervix cancer brachytherapy - 3D dose volume parameters and aspects of 3D image-based anatomy, radiation physics, radiobiology. Radiother Oncol 2006; 78: 67-77.

12. Pham H, Chen $\mathrm{Y}$, Rouby E et al. Changes in high-dose-rate tandem and ovoid applicator positions during treatment in an unfixed brachytherapy system. Radiology 1998; 206: 525-531.

13. Tanderup K, Hellebust TP, Lang S et al. Consequences of random and systematic reconstruction uncertainties in 3D image based brachytherapy in cervical cancer. Radiother Oncol 2008; 89: 156-163.

14. Grigsby PW, Georgiou A, Jeffrey F et al. Anatomic variation of gynecologic brachytherapy prescription points. Int J Radiat Oncol Biol Phys 1993; 27: 725-729.

15. Bou-Zeid W, Bauer C, Kim Y et al. Clinical validation of a real-time applicator position monitoring system for gynecologic intracavitary brachytherapy. Biomed Phys Eng Express 2016; 2: 045008 .

16. Schindel J, Zhang W, Bhatia SK et al. Dosimetric impacts of applicator displacements and applicator reconstruction-uncertainties on 3D image-guided brachytherapy for cervical cancer. J Contemp Brachytherapy 2013; 5: 250-257.

17. Karlsson L, Thunberg P, With A et al. 3D image-based adapted high-dose-rate brachytherapy in cervical cancer with and without interstitial needles: measurement of applicator shift between imaging and dose delivery. J Contemp Brachytherapy 2017; 9: 52-58.

18. Dumane V, Yuan Y, Sheu R et al. Computed tomographybased treatment planning for high-dose-rate brachytherapy using the tandem and ring applicator: influence of applicator choice on organ dose and inter-fraction adaptive planning. J Contemp Brachytherapy 2017; 9: 279-286.

19. Yin G, Wang P, Lang J et al. Dosimetric study for cervix carcinoma treatment using intensity modulated radiation therapy (IMRT) compensation based on 3D intracavitary brachytherapy technique. J Contemp Brachytherapy 2016; 8: 221-232.

20. Carvalho H, Mendez L, Stuart $S$ et al. Implementation of image-guided brachytherapy (IGBT) for patients with uterine cervix cancer: a tumor volume kinetics approach. J Contemp Brachytherapy 2016; 8: 301-307.

21. Soliman A, Owrangi A, Ravi A et al. Metal artefacts in MRIguided brachytherapy of cervical cancer. J Contemp Brachytherapy 2016; 8: 363-369.

22. Kim Y, Huang Y, Chesnut D et al. Applicator Displacements Due To Additional Scan For 3D Image-Guided Brachytherapy For Cervical Cancer. Radiother Oncol 2011; 99: S257-S258.

23. Tanderup K, Pötter R, Lindegaard JC et al. PTV margins should not be used to compensate for uncertainties in $3 \mathrm{D}$ image guided intracavitary brachytherapy. Radiother Oncol 2010; 97: 495-500. 